Running Head: EXERGAMES

Effects of Interactive Physical-Activity Videogame Training on Physical and Cognitive Function in Older Adults

\author{
Pauline Maillot and Alexandra Perrot \\ Université Paris-Sud \\ Alan Hartley \\ Scripps College
}

Author's note

Pauline Maillot, Alexandra Perrot, CIAMS EA 4532, Université Paris-Sud; Alan Hartley, Scripps College, Claremont, California.

Correspondence concerning this article should be addressed to Pauline Maillot, UFR STAPS - CIAMS, Bât 335, Université Paris-Sud, 91405 Orsay Cedex, France.

Email: pauline.maillot@u-psud.fr. 


\begin{abstract}
The purpose of the present study was to assess the potential of exergame training based on physically-simulated sport play as a mode of physical activity that could have cognitive benefits for older adults. If exergame play has the cognitive benefits of conventional physical activity and also has the intrinsic attractiveness of videogames, then it might be a very effective way to induce desirable lifestyle changes in older adults. To examine this issue, the authors developed an active video game training program using a pretest-training-post test design comparing an experimental group $(24 \mathrm{x} 1 \mathrm{hr}$ of training) with a control group without treatment. Participants completed a battery of neuropsychological tests, assessing executive control functions, visuospatial functions, and processing speed functions to measure the cognitive impact of the program and a battery of functional fitness tests in order to measure the physical impact of the program. The trainees improved significantly in measures of game performance. They also improved significantly more than the control participants in measures of physical function and cognitive measures of executive control and processing speed functions, but not on visuospatial measures. It was encouraging to observe, with a physicallysimulated sport games, transfer benefits to cognitive and physical skills that are directly involved in functional abilities needed by older adults in everyday living (e.g., Hultsch, Hertzog, Small, \& Dixon, 1999).
\end{abstract}

Key Words: physical activity, aging, video game, cognition, exergames 
Effects of Interactive Physical-Activity Videogame Training on Physical and Cognitive Function in Older Adults

A ubiquitous observation in studies of human aging is that cognitive abilities differ between young and elderly adults in a number of neurocognitive domains such as reasoning, attention, memory, and processing speed (e.g., Craik \& Salthouse, 1999; Hoyer \& Verhaeghen, 2006; Park \& Gutchess, 2002; Salthouse, 2004). Nevertheless, epidemiological work suggests that in old age a lifestyle rich in mental, physical, and social stimulation could have beneficial influences on the level of cognitive performance (e.g., Hultsch, Hertzog, Small, \& Dixon, 1999; Kramer, Bherer, Colcombe, Dong, \& Greenough, 2004). Generalized transfer is relatively rare in studies of learning but there is clear evidence that regimens involving physical activity training or video game playing can result in improved cognitive function in older adults (e.g., Green \& Bavelier, 2008; Green, Li, \& Bavelier, 2010; Spence \& Feng, 2010). Exercise adherence is a particular challenge with older adults (Chao, Foy, \& Farmer, 2000), but video game playing is increasingly popular among middle-aged and older adults (e.g., Flew \& Humphreys, 2005). Products that have recently become available support entertaining video games that combine game play with significant physical exercise by using physical input devices (e. g., Nintendo Wii and Microsoft Xbox 360 Kinect). We will call these exergames. There has been a rapid growth of the popularity of exergames, including among the elderly (e.g., Vance, McNees, \& Meneses, 2009). The purpose of the present study was to assess the potential of exergame training based on physically-simulated sport play as a mode of physical activity that could have cognitive benefits for older adults. If exergame play has the cognitive benefits of conventional physical activity and also has the intrinsic attractiveness of videogames, then it might be a very effective way to induce desirable lifestyle changes in older adults.

Physical Activity and Cognition 
The relationship between physical activity and cognition has been examined empirically with cross-sectional designs (e.g., Clarkson-Smith \& Hartley, 1989; Hillman et al., 2006; Perrot, Gagnon, \& Bertsch, 2009; Shay \& Roth, 1992) and with physical training interventions (e.g., Dustman et al., 1984; Hawkins, Kramer \& Capaldi, 1992; Kramer et al., 1999). It has also been approached from neuroscience perspectives (Colcombe, Kramer, McAuley, Erikson, \& Scalf, 2004; Erickson \& Kramer, 2009). The results of meta-analytic reviews have established that the beneficial effects of physical activity participation on cognitive performance in older adults are reliable, with one review reporting an effect size (ES, Cohen, 1988) that was moderate in size $(E S=0.48$ using Hedges's (1982) formula; Colcombe \& Kramer, 2003) and another reporting a small to large effect ( $E S=0.10$ to 1.17; using the Weighted Mean Difference and Standardised Mean Difference; Angeraven, Aufdemkampe, Verhaar, Aleman, \& Vanhees, 2008). Additionally, Colcombe and Kramer indicated that larger effects of aerobic exercises $(E S=0.41)$ and combined training (aerobic with strength exercises; $E S=0.59)$ were evident for executive function tasks $(E S=0.68)$ than for either processing speed tasks $(E S=0.27)$, visuospatial-awareness tasks $(E S=0.43)$, or controlled cognitive tasks $(E S=0.46)$. Empirical studies have largely focused on aerobic exercise, based on the presumed role of cardiovascular fitness as a potential mechanism, in the relationship between cognitive decline and physical activity (e.g., Chodzko-Zajko, 1991; Colcombe et al., 2004; Kramer et al., 1999). However, findings from the meta-analytic reviews suggest that cardiovascular fitness is not the only mechanism of the effect (Angeraven et al., 2008; Colcombe \& Kramer, 2003; Etnier, Nowell, Landers, \& Sibley, 2006). This then allows the possibility that increases in cardiovascular fitness are not necessary for cognitive benefits, and, consequently other forms of physical activity that do not particularly emphasize improvements in cardiovascular fitness should be explored for their possible cognitive benefits. As we noted, there is also an underlying issue. Although the 
effects of physical activity on cognitive vitality are widely recognized in popular culture, resistance to exercise among the elderly remains very high (U.S. Department of Health and Human Services, 1996). This is presumed to be due to the unattractiveness and inaccessibility of physical activity as well as to functional restrictions (i.e., reduced cardiorespiratory endurance, reduced resistance strength, weight gain) related to advancing age (e.g., Hughes, Seymour, Campbell, Whitelaw, \& Bazarre, 2009; Schutzer \& Graves, 2004).

\section{Video Games and Cognition}

Training regimens based on sedentary video games can improve cognitive skills (e.g., Achtman, Green, \& Bavelier, 2008; Green \& Bavelier, 2008; Green et al., 2010). The benefits of video games for cognitive performance have been demonstrated in younger adults primarily in cross-sectional studies comparing game players with non-game players on visuospatial attentional functions (for a review, see Green \& Bavelier, 2006) and on attention, memory, and executive control functions (Boot, Kramer, Simons, Fabiani, \& Gratton, 2008). In older adults, it has been observed that after several weeks of video game training elderly game players showed improvements when compared with control groups on reaction time (e.g., Clark, Lanphear, \& Riddick, 1987; Goldstein et al., 1997), manual dexterity (Drew \& Waters, 1986), visuomotor coordination (Dustman, Emmerson, Steinhaus, Shearer, \& Dustman, 1992) and executive control functions such as task switching, working memory, visual short-term memory and reasoning (Basak, Boot, Voss, \& Kramer, 2008). However, there are reasons to question whether there is general transfer of video game play to cognitive domains. Ackerman, Kanfer and Calderwood (2010) had a sample of adults between the ages of 50 and 71 complete 20 1-hr training sessions over the course of one month using the Nintendo Wii Big Brain Academy, a completely sedentary activity. The results indicated substantial improvements on the Wii tasks themselves, but there was no significant transfer of training from the Wii practice to more measures of fluid and crystallized intelligence and 
perceptual speed abilities, administered before and after the month of cognitive training activities. Although a positive relation between video game training and cognition has been demonstrated, it would seem that the transfer of the video game training to measures of cognition is not systematic, but rather may depend on several aspects of the training such as task difficulty, motivation and arousal, feedback, and variability (cf., Green \& Bavelier, 2008). Moreover the type of video games employed could have an important effect on whether transfer of training is observed. For example Basak et al. (2008) did not obtain the same improvements for visuospatial skills using a real-time strategy game that Green and Bavelier $(2003,2006)$ had obtained with action video games. The characteristics of the game itself could be directly related to the types of processes that are modified by playing the game (e.g., Achtman et al., 2008) meaning that transfer is not as general as claimed. The results of previous training programs using video games provide us with no clear guidance for predictions in the present study. It is possible that video game training results in general transfer only when physical activity is involved.

\section{Exergames and Cognition}

Exergames, like the sports that they simulate, make both physiological demands for increased energy expenditure (Graves et al., 2010) and cognitive demands (Quiroga et al., 2009). For example, both in tennis and in a simulation such as Wii Tennis, one must run to the ball and strike it and, at the same time, calculate where to place the return for maximum advantage. We know that physical exercise has broad cognitive benefits, perhaps even if cardiovascular fitness is not improved. Video game playing may have cognitive benefits, but even it if does not, it is highly motivating and likely to promote exercise adherence. We are aware of only two studies using exergames. Rosenberg et al. (2010) found a reduction in depressive symptoms and improvement in mental health-related quality of life and in a measure of global cognitive functioning after 35 min session three times weekly for 12 weeks 
although the critically important untrained control group was not included. Nitz, Kuys, Isles, and Fu (2009) found improved balance and lower limb muscle strength after 30 min sessions twice weekly for 10 weeks. To our knowledge, no study has examined the impact of exergames on cognitive functioning in older adults.

\section{The Present Study}

The purpose of the present study was to determine whether exergame training in physically-simulated sport activity would show transfer to cognitive functions in older adults. To examine this issue, we developed an active video game training program using a pretesttraining-post test design comparing an experimental group $(24 \mathrm{x} 1 \mathrm{hr}$ of training) with a control group without treatment. We selected the Nintendo Wii gaming system owing to its accessibility for novice and older populations, the relevance of the games to actual sport, and its ready availability in the marketplace of commercial software. In order to maximize the physiological challenge and keep the spirit of physical activity, only games of physicallysimulated sport were selected on this exercise program: Wii Sports, Wii Fit and Mario \& Sonic on Olympic Games.

We tested two main hypotheses. The first was that exergame training would result in improved performance on the Wii sports tasks themselves. The second was that there would be practice-related improvements on a wide range of neuropsychological tests, including executive control tasks, visuospatial tasks and processing speed tasks. On the basis of previous findings of transfer effects for physical activity, we expected that the exergames program would lead the benefits on measures of executive control (e.g., Colcombe \& Kramer, 2003; Kramer et al., 1999). Further, to the extent that exergames make mental demands similar to those of sedentary video games we expected, on the basis of the findings of practice effects for video games, that performance on visuospatial tasks (e.g., Green \& Bavelier, 2003, 2006) and on processing speed tasks (e.g., Clark, Lanphear, \& Riddick, 1987; Drew \& 
Waters, 1986; Dustman et al., 1992; Goldstein et al., 1997) would be enhanced. Because we were not certain that Wii games would be sufficiently challenging to produce a change in cardiovascular fitness, we did not make a prediction about changes in physical function.

\section{Method}

\section{Participants}

Potential participants were contacted through flyers posted in town halls and community senior centers. People responding were contacted by phone and they provided an estimation of their physical activity, video game use and health status. All potential participants also rated their health on a 5 -point health scale $(1=$ very bad, $2=$ bad, $3=$ fair, 4 = good, $5=$ excellent), and those who rated their health as very bad or bad were excluded. We included only those individuals who reported never playing video games and living a sedentary lifestyle. We based our selection criteria on the recommendations of several health guides (including the Canadian Health Network in Canada and the Institut National de Prévention et d'Education pour la Santé in France).

Thirty-two independently-living older adults ( 27 women, 5 men, between 65-78 years) recruited from the region of Paris, France, volunteered to take part in this longitudinal intervention study. All participants were of European, Caucasian origin and were native French speakers. They gave their informed consent, and were not compensated for their participation. Each participant was required to have a medical certificate permitting the practice of physical activity. All participants were right-handed and had normal or correctedto-normal vision and audition. The participants completed the Mini-Mental Status Examination (Folstein, Robins, \& Helzer, 1983), the Geriatric Depression Scale (Yesavage et al., 1982-1983), and the Modifiable Activity Questionnaire (MAQ, Kriska et al., 1990) which assesses current (past-year) physical activity for different activity categories practiced at least 10 times per year. By random assignment, 16 participants were assigned to the exergames 
training group and 16 other participants were assigned to a no-training no-contact control group. Over the course of the study, one participant in the training group gave up on the third training session reporting that it was physically too difficult. One control participant could not attend the post test session owing to medical problems unrelated to the study. Accordingly, the analysis of results of this study was based on the 15 individuals remaining in each group. Demographic information for both groups is listed in Table 1. There were no significant differences between the two groups in age, years of education, body mass index, or scores on the MMSE, GDS, and MAQ.

\section{Apparatus}

For the exergame training we used the Nintendo Wii, a video game console with motion-sensitive technology. The Wii Remote and the Nunchuk, the primary wireless controllers for the console, use a combination of built-in accelerometers and infrared detection to sense their positions in three-dimensional space when pointed at LEDs within the console's Sensor Bar. This design allows game players to control their avatars (graphical representations of the user) using physical gestures as well as traditional button presses. We used also the Nintendo Wii Balance Board which has a similar shape to a body-weight scale, with a flat rectangular platform $51.1 \mathrm{~cm}$ wide by $31.6 \mathrm{~cm}$ long by $5.3 \mathrm{~cm}$ deep. Four force sensors record the weight of the player and the trajectory of the center of pressure of the participant during the game. It communicates wirelessly with the Nintendo Wii video game console. The video game display was a LCL projector (Panasonic Model PT-AX200E) projecting on a portable screen (Epson ELPSC06) $76 \mathrm{~cm}$ in height and $102 \mathrm{~cm}$ in width.

\section{Measures}

\section{The Functional Fitness Battery}

The physical impact of training program was evaluated by performance on a functional fitness battery, by heart rate measures (in beats per minute, bpm), and by ratings of 
perceived effort. The functional fitness battery measures the physiologic capacity to perform normal everyday activities safely and independently without undue fatigue (Rikli \& Jones, 1999). We used the Senior Fitness Test (SFT, Rikli \& Jones, 2001) which measures the underlying physical parameters associated with functional ability and which identifies whether an older adult may be at risk for loss of ability to perform functional movements such as walking, stair climbing, and standing up. The SFT protocol comprises seven components completed in 30 to $40 \mathrm{~min}$ following a $5 \mathrm{~min}$ warm-up exercise. Height (m) and body weight (kg) were measured in order to calculate Body Mass Index (BMI, $\mathrm{kg} / \mathrm{m}^{2}$ ). The chair-stand test measures muscle strength for lower body, and the arm curl test measures upper body strength. The 6-Minute Walking Test (6MWT) evaluates cardio-respiratory fitness. The number of meters walked, the maximum heart rate, the mean heart rate, and the rated exertion using the modified Borg (1982) scale (range 0 to 10 , with $0=$ complete rest and $10=$ extremely strong) were recorded. The chair sit-and-reach test evaluates lower body flexibility and the back scratch test measurement upper body flexibility. The 8-foot up-and-go test evaluates agility and balance. Rikli and Jones (2001) give a complete description of these tests ${ }^{1}$. Heart rate (HR) was monitored using a standard chest lead connected to a digital pulse transmitter (SUUNTO T6 - software SUUNTO Training Manager, version 2.3.0.15). Several recordings were made during the study: at the outset of the study, during the 6MWT during the pretraining and post-training sessions (walk and rest), and for the training group, during the second, twelfth, and twenty-second training sessions. The resting heart rate (RHR) was measured for 15 minutes with participants in the supine position at the first assessment session. The purpose was to be able to quantify the intensity of the physical exercise throughout the course of the program through the calculation of the proportion of the heart rate reserve according to the method of Karvonen (1957). Heart rate reserve (HRR) is the difference between the estimated maximum heart rate $(\mathrm{MHR}=205-0.685 \mathrm{x}$ Age $)$ and the 
RHR. There are various formulas to estimate MHR; this one is recommended as the most accurate univariate estimate in a thorough review by Robergs and Landwehr (2002). For any exercise bout with heart rate $H R_{E}$, the proportion of $\mathrm{HRR}$ used is calculated as $\left(\mathrm{HR}_{\mathrm{E}}-\right.$ RHR)/HRR. Finally at end of the 6MWT and after every training session, participants were asked to score their perceived exertion using the Borg scale.

\section{The Cognitive Battery}

The battery included a number of tasks that fell into three categories: executive control tasks (Trail Making test, Stroop Color Word Interference test, Letter Sets test, Matrix Reasoning test ${ }^{2}$, Digit Symbol Substitution test), visuospatial tasks (Spatial Span test, Directional Headings test, Mental Rotation test) and processing speed tasks subdivided into two categories, perceptual speed (Cancellation test and Number Comparison test) and psychomotor speed (the Reaction Time test and Plate Tapping test). All these tasks are described below.

\section{Executive Control Tasks}

Trail Making test. The Trail Making test (Corrigan \& Hinkeldey, 1987), originating from the Army Individual Test Battery, is a timed paper-and-pencil task that consists of two separate parts. Part A involves drawing a line to connect consecutive numbers (i.e., from 1 to 2 to 3 and so on up to 25). Part B requires the participant to connect numbers and letters in an alternating progressive sequence (i.e., 1 to $\mathrm{A}$ to 2 to $\mathrm{B}$ and so on). Without lifting their pencil from the paper, participants began at number 1 and connected the circles in the instructed order as quickly as possible without making any errors. If an error was made, the experimenter indicated the error and allowed the participant to correct the mistake and continue the task. The measure is the time to complete. Because Part A of the Trail Making Test has been viewed as primarily a measure of speed, the measure we used in our analyses 
was the time for Part B less the time for Part A. Following Corrigan and Hinkeldey (1987), we reasoned that the difference would be a purer measure of executive function.

Stroop test. The Stroop test (Stroop, 1935), is a frequently used measure of executive function; we used a modified version (Bohnen, Jolles, \& Twijnstra, 1992; Chatelois et al., 1996). Participants are required to verbally identify the color of the stimulus in each of four conditions. In the congruent color-naming condition, the stimulus is string of symbols printed in colored ink, such as a series of red Xs. In the color-reading condition, the stimulus is a color name written in black ink. In the incongruent condition, the stimulus is a color name printed in a different ink color, such as the word "blue" printed in red ink. Finally, in the flexibility condition, the stimuli are the same as in the congruent condition except that the participant reads the color word rather than the ink color if the stimulus has a border. In all conditions, the number of correct answers given in $45 \mathrm{sec}$ was the measure of performance. Color-naming and color-reading conditions are task-training conditions that were not included in the analysis. Only the incongruent and flexibility conditions were used as measures of the executive functioning.

Letter Sets test. Participants were given 15 items each containing five sets of letters (e.g., NOPQ DEFL ABCD HIJK UVWX) and had to choose the set that did not follow the rule defined by the four other sets. The Letter Sets test was taken from the Educational Testing Service (ETS) Kit of factor-referenced cognitive tests (Ekstrom, French, Harman, \& Dermen, 1976). The score was the number of items correctly completed in 13 minutes.

Matrix Reasoning test. This test consists of a sequence or group of designs, in which the individual is required to choose the one design from a number of choices that correctly completes the sequence. This test is a subtest of Wechsler Abbreviated Scale of Intelligence (1999) and measures abstract nonverbal reasoning ability. The score was the number of items correctly completed. 
Digit Symbol Substitution test. Participants were provided with key matching digits to various symbols and were asked to write in the appropriate symbol for each digit to several rows of digits. The score was the number of correctly coded digits completed in $120 \mathrm{sec}$. The Digit Symbol Substitution test originated from the WAIS-R (Wechsler, 1997a). Visuospatial Tasks

Spatial Span test. The Spatial Span test is a subtest of the Weschler Memory Scale (Wechsler, 1997b). The apparatus consists of ten blue wooden blocks $3.1 \mathrm{~cm}$ square fixed to a $20 \mathrm{~cm}$ by $25 \mathrm{~cm}$ white, wooden board. The blocks are numbered on the sides facing the examiner as a means of facilitating administration and recording of the path sequences. The blocks are tapped in a predetermined sequence by the examiner using the eraser end of a pencil at the rate of one block per second, and participants are required to reproduce the block-tapping sequences when the stimulus sequence is completed by touching the appropriate blocks. This procedure continues with increasing numbers of blocks touched until the participant cannot correctly reproduce the sequence. The score is the largest number correctly reproduced. The backward Spatial Span test is exactly the same except that the participant must reproduce the sequence in reverse order.

Directional Headings test. This is a speeded paper and pencil test of spatial ability (Cobb \& Mathews, 1972). Each item comprises three pieces of information that reflect cardinal points on a compass (e.g., the letter "W", a symbol "๕", and the notation "270", each denote "West"). The individual is asked to determine the direction indicated (if all information is consistent), or indicate that the information is inconsistent. The score is the number of correct items in $60 \mathrm{sec}$.

Mental Rotation test. The mental rotation test is a variant of the Shepard and Metzler figures (1971) introduced by Vandenberg and Kuse (1978) who used the original figures to create a pencil and paper test for spatial abilities. The 20 figures are presented as two- 
dimensional visual images that are constructed of 10 cubes and are perceived as three dimensional objects. The task for subjects is to decide, quickly and accurately, whether a pair of two figures shows the same object or different objects. The score is the number of items correct in $10 \mathrm{~min}$.

\section{Processing Speed Tasks}

Cancellation test. This is a perceptual speed test (Ackerman, 1990) consisting of a page of randomly-generated uppercase English letters. All letters occurred with equal probability and participants were instructed to place a line through each occurrence of a target letter (A) in 60 seconds. The score was the number correctly marked.

Number Comparison test. This is another perceptual speed test (Ackerman \& Cianciolo, 2000) consisting of two 50-item columns of digits. Participants are instructed to place a check mark between adjacent numbers that match exactly. The score is the number of correct items in 90 seconds.

Reaction Time test. Speed of response in milliseconds was measured for simple reaction time (SRT; a single stimulus) and choice reaction time (CRT; four stimuli). Both SRT and CRT can be decomposed into decision time (DT; the time to lift a finger from a start button to begin movement toward to a target button) and Movement Time (MT; the time from lifting the finger until the target button is pressed). Instructions and stimuli were presented with a Pentium 4 PC, with standard keyboards and E-Prime software which recorded accuracy and response time (version 1.1 beta 1.0, Schneider, Eschman, \& Zuccolotto, 2002). The stimulus was a red circle. For SRT the red circle appeared only at the left corner of the screen and the participant responded by pressing the spacebar as quickly as possible. For CRT the red circle could appear at one of four positions along an imaginary circular arc. The participant responded by pressing the a key on the numeric keypad corresponding to the position of the red circle- 1 for lower left, 7 for upper left, 9 for upper right, and 3 for lower 
right. Performance was measured as the mean SRT and CRT in milliseconds on a block of 12 trials.

Plate Tapping test. The Plate Tapping test, a part of the Eurofit Testing Battery, is a response speed test using an alternating tapping action which measures upper body reaction time, hand-eye quickness, and coordination (Bovend'eerdt, Kemper, \& Verschuur, 1980). Two green discs (20 cm diameter) are placed with their centers $60 \mathrm{~cm}$ apart on a table and a rectangle $(30$ by $20 \mathrm{~cm}$ ) is placed equidistant between both discs. One hand is placed on the rectangle. The subject moves the other hand back and forth over the hand in the middle alternately tapping the two discs as quickly as possible. This action is repeated for 30 seconds. The score is the number of taps. Participants had two trials with each hand, the mean scores were recorded.

\section{Procedure}

The entire study spanned 14 weeks. This included the pre-training (first week) and post-training (fourteenth week) sessions for assessment and, for the training group, the training sessions. Participants completed a battery of neuropsychological tests, assessing executive control functions (five tests with a total of six measures), visuospatial functions (three tests with a total of four measures), and processing speed functions (four tests with a total of eight measures), in order to measure the cognitive impact of the program and a battery of functional fitness tests in order to measure the physical impact of program. Each time, the cognitive battery and the functional fitness batteries were administered over two days within one week, with a 90 minute session each day. The 12 cognitive assessments tasks were presented in the following fixed order: On the first day, performance on executive control and processing speed tasks was assessed; on the second day, performance on visuospatial tasks and functional fitness were assessed. 
Participants in the training group completed two 1-hr exergame sessions per week over a period of 12 weeks, resulting in total training time of $24 \mathrm{hr}$. Each participant was paired with another for each session with the intention of making the exergame playing more enjoyable and motivating adherence to the regimen. Participants were asked not to play exergames, except for this study. Each session began with a warm-up and finished with a cool-down in order to reduce the risk of injury (e.g., Jones \& Hammig, 2009).

Each training session was divided into three periods. During the first period, participants played in pairs at the Wii Tennis or Wii Boxing game alternating each session. Only on the first, twelfth and twenty-fourth sessions, they played at the Wii Bowling game. During the second period, participants took turns using the balance board to play the Wii Soccer Headers, the Wii Ski Jump, and the Wii Marbles games. During the final period, participants played four games on which we recorded performance: Wii Ski Slalom, Wii Hula Hoop, Wii Trampoline, and Wii Tennis Return of Serve. We classified each of these four tasks based on the primary skill required: The Ski Slalom game requires balance; the Hula Hoop game requires energy and stamina; the Trampoline game requires cognitive judgment; and the Tennis game requires a variety of skills so we classified it as global.

For the balance task (Ski Slalom), the participants "slalomed" by shifting their weight to alternate between blue and red doors as quickly as possible. The performance score is a function of the time to complete the course and the numbers of door cleared. For the energy task (Hula Hoop), the score was the number of successful rotations of the hoop in $70 \mathrm{sec}$. For the cognitive task (Trampoline), the participants achieved up to 10 points by making the indicated movements for optimal control of the avatar. For the global task (Tennis Return of Serve), the score was the number of serves from the computer's ball machine successfully returned before an error occurred. 
Each training session was supervised by a physical trainer. The coach's functions were (a) to implement the training schedule and give any necessary explanations, (b) to ensure the safety of the participants and particularly to prevent the risk of fall, (c) to prevent participants from using incorrect postures or movements or cheating movements, and (d) to give participants feedback on their performance and note their exercise adherence. The participants were urged to try to increase the level of challenge and to improve their performance on each activity over the course of the training. At the end of the training program, the participants completed a questionnaire that measured their subjective impression of the program. Six questions were asked, two in the form of 5-point scales on the similarity between the exergame session and conventional forms of physical activity $(1=$ very much, $2=$ much, $3=$ relatively, $4=$ not much, $5=$ none $)$, and on the difficulty of the training sessions $(1=$ very easy, 2 = easy, 3 = reachable, 4 = difficult, $5=$ very difficult), and four other yes-no questions on whether their body knowledge had improved, whether they would like to continue an exergames practicum, whether they would like to begin a program of physical activity, and whether they would like to acquire an exergame game console.

The participants in the control group committed themselves not to modify their sedentary lifestyle and not to begin playing video exergames games or engage in other physical activity over the 14 weeks of the study.

\section{Results}

The first step in the analysis was to verify that the training and control groups were equivalent at the outset of the program. T tests were used to compare the pre-training scores on both physical and psychological measures for those who would later be assigned to the training and control groups. The descriptive and inferential statistics are given in Table 2. Among the 35 comparisons, only one was significant. Those who would be assigned to the training group rated the 6-min walk as more taxing than those who would be assigned to the 
control. Because there was only this one difference and that in a subjective assessment, we deemed the two groups equivalent.

The second step in the analysis was to characterize the participants in the training group and the nature and effect of their training. The number of completed sessions for the 15 participants ranged from 20 to $24(\mathrm{M}=23.40)$. Overall adherence was $97.50 \%$, with 351 out of a possible 360 sessions ( 15 participants for 12 weeks with 2 sessions per week) completed by participants. The final questionnaire indicated that the exergame training was manageable for older persons (80\% agreed), and seemed comparable to other physical activity $(80 \%$ of results ranged between very much and relatively). All participants reported that they would like to continue with exergame activity, however only $40 \%$ contemplated acquiring a game console. Finally, $67 \%$ of participants thought that their body knowledge improved with training program, and $47 \%$ were considering beginning a program of physical activity. Participants showed significant improvement in performance across training sessions on all four of the exergames: for the balance task, $F(23,207)=10.79, p<.01, \eta^{2}=.55$, for the cognitive task, $F(23,207)=14.25, p<.01, \eta^{2}=.61$, for the energy task, $F(23,138)=2.66, p$ $<.01, \eta^{2}=.31$, and for the global task, $F(23,230)=6.70, p<.01, \eta^{2}=.40$. Follow up tests using Tukey's HSD, showed significant improvement over the first session by the sixth session in the balance task (Ski Slalom), by the seventh session in the cognitive task (Trampoline), by the tenth session in the global task (Tennis Return of Serve), and by the twenty-third session in the energy task (Hula Hoop). We note that only six of the participants continued the energy task until the final training session. In addition, heart rate was measured during the second, twelfth, and twenty-second training sessions. There were no significant changes across these three sessions in mean heart rate, $F(2,26)=0.78, p=.47$, in maximum heart rate, $F(2,26)=1.97, p=.16$, or in proportion of heart rate reserve achieved, $F(2,26)=$ $2.00, p=.16$ 
The central step in the analysis was to determine whether the training regimen resulted in greater change in the trained group than the untrained controls. We calculated the change score by subtracting the pretest score from the posttest score for each measure. First, we explored the possibility of test-retest improvement in the control group using one-group $t$ tests to test the observed change against zero. There was no significant change. In order to determine whether the change was significantly greater in the trained group than in the controls we used the following approach: First, we carried out a MANOVA with all the measures within a set as dependent variables; then, if the overall difference between treatment and control was significant, we carried out $t$ tests on each of the component measures, using the modified Bonferroni procedure to protect against an inflated chance of a Type I error (Holm, 1979). The first set of comparisons was for measures of physical performance. The descriptive and inferential statistics for those change scores are given in Table 3. Physical improvement was significantly greater in the treatment group, Wilk's $\Lambda=.31, F(10,18)=$ 4.06, $p=.005, \eta^{2}=.693$. The Borg Scale judgments of exercise intensity for the 6-min walk, which are subjective measures, were omitted from this analysis. Follow-up tests showed that greater improvement in the training group than in the controls was observed for all the physical measures except the Back-Scratch measures of flexibility. With unprotected tests, there were significant differences on three of the four flexibility measures. There was also no significant difference in the Borg Scale ratings. The second set of comparisons was for measures of processing speed, for which descriptive and inferential statistics are given in Table 4. The effect of group was significant, Wilk's $\Lambda=.21, F(8,21)=9.75, p<.001, \eta^{2}=$ .788. Follow-up tests showed that improvement for all of these measures was significantly greater in the training group than in the control group. The third set of comparisons was for measures of visuospatial function, for which descriptive and inferential statistics are given in Table 4. The difference between the treatment and control groups was not significant, Wilk's 
$\Lambda=.77, F(4,25)=1.87, p=$ n.s, $\eta^{2}=.230$. The fourth set of comparisons was for measures of executive function, for which descriptive and inferential statistics are also given in Table 4. Here the improvement was significantly greater in the treatment than in the control group, Wilk's $\Lambda=.19, F(6,23)=15.79, p<.001, \eta^{2}=.805$, and follow-up tests showed that this was true for all of the measures.

\section{Discussion}

The purpose of the present study was to determine the potential of training based on physically-simulated sport play as a mode of activity that could have cognitive benefits for older adults. The training program was modeled on programs of physical activity and programs of sedentary videogame play that had previously shown cognitive benefits.

The first hypothesis was that exergame training would produce gains in performance on the Wii sports tasks themselves. We found that practice did result in improved performance on the Wii tasks. Participants showed significant and substantial gains in performance on the four performance tasks which challenged a variety of abilities over the course of 24 hours of practice across 12 weeks. These improvements showed that this inactive population could benefit from the stimulation of exergames. This finding is strengthened by both the high levels of exercise adherence $(97.50 \%)$ and the questionnaire reports that participants appreciated and would like to continue exergame activity. Graves et al. (2010) showed that Wii tasks generated more enjoyment than sedentary videogame or treadmill exercise in older adults. Our results underscore the potential of exergames in terms of accessibility and intrinsic attractiveness for older adults. Thus interactive physical-activity videogames could be an enjoyable alternative to physical activity (e.g., Hughes et al., 2009; Schutzer \& Graves, 2004).

The second hypothesis of this study was that interactive physical-activity videogame training was a mode of activity that could have cognitive benefits for older adults. First, we 
expected a range of practice-related improvements on cognition as previously found in programs of physical activity. In fact, the results demonstrated significant benefits of exergame training on executive control and processing speed tasks from before to after training. Out of six measures of executive functions, we found significant transfer of exergame training on all measures $\left(\eta^{2}=0.80\right)$. Out of eight measures of processing speed, all measures showed significant improvement $\left(\eta^{2}=0.79\right)$. This large beneficial effect of training on cognition is in line with findings of many empirical studies on aerobic exercise (e.g., Dustman et al., 1984; Hawkins, Kramer \& Capaldi, 1992; Kramer et al., 1999). The results are also consistent with the meta-analytic review of Colcombe and Kramer (2003) which indicated a large effect of physical exercise on executive function tasks $(E S=0.68)$, which it has been argued are particularly sensitive to the effects of aging (West, 1996). They also found positive but somewhat smaller effects of physical activity on processing speed tasks $(E S=0.27)$

Dustman and collaborators $(1984,1992)$ compared the cognitive benefits in older adults among several groups who had followed different training regimens (two of which were aerobic exercise and sedentary videogame playing). The aerobic exercise training showed more transfer of training to executive control and processing speed tasks than did videogame training. They hypothesized that the important difference between sedentary videogame playing and aerobic fitness training may be the improvement in oxygen transport and utilization that results from aerobic fitness. Dustman and collaborators $(1984,1992)$ suggested that the increased availability of oxygen for cerebral metabolic activity may be responsible for the improved neurocognitive performance that occurs with fitness training. Consistent with this hypothesis, our results showed significant effects of training on physical status, and more particularly on cardio-respiratory fitness (e.g., 6MWT). Furthermore, the mean heart rate measured during exergame playing was $102.5 \pm 7.9$ beats/min, which 
corresponded to $41.5 \pm 9.48 \%$ of the estimated heart rate reserve (HRR). This level of HRR is within the American College of Sports Medicine (ACSM, 2006) recommended "moderate" intensity range of 40-59 \% HRR (e.g., Howley, 2001). Therefore, we speculate that the improvements in cognitive performance with exergame training are in part due to the gains in cardiovascular fitness achieved through regular physical activity, which increased the ability of the heart to deliver oxygen to working muscle and which is indicative of an increase in cardiovascular fitness. So, in this view our results generated positive benefits on cognition in the same manner as physical activity programs. However, one limitation of this interpretation is that we are simply comparing our results with the existing empirical literature rather than including a conventional treatment group in our study which included only physical activity and not exergames. It would be necessary to carry out another intervention study that included a physical activity training group in order to determine how much of the exergame benefits are due to the physical activity.

Although the exergame play engenders a significant increase of energy expenditure (e.g., Graves et al, 2010), a recent study on the cardiovascular and metabolic responses to Wii Fit videogame playing found differences among the Wii games in the HRR response (Guderian et al., 2010). Moreover, although for many of our participants HRR response fell within the ACSM recommended "moderate" intensity range, it is also true that for some participants HRR response were below that level. Thus the exercise intensity of Wii exergame playing may not be sufficient to explain the improvements on executive control and processing speed tasks. We speculate that some other aspects of the activity may also account for portions of the improvements we observed. Indeed, the intrinsic characteristics of exergames, such as the motivation and arousal, task difficulty, or feedback (Green \& Bavelier, 2008), may well be partly responsible for cognitive improvements as we will discuss below. 
The positive benefits on executive control and processing speed performance that we have found with exergames training are also consistent with benefits previously reported with sedentary videogame training. Consistent with the results of Basak et al. (2008), in which training on a strategy-based game was found to improve executive function, we found evidence for transfer on executive control tasks. Our results also showed a significant transfer of training to processing speed tasks. Other studies using sedentary videogames have reported a robust benefit specifically on psychomotor speed, especially reaction time (e.g., Clark et al, 1987; Drew \& Waters, 1986; Dustman et al., 1992; Goldstein et al., 1997). Our participants showed improvement not only for measures of psychomotor speed (e.g., tapping) but also for measures of perceptual speed (e.g., cancellation). Perceptual speed is the ability to rapidly and accurately search, compare, and identify elements presented side by side or separated in the visual field. To our knowledge, this is the first time that a positive effect on perceptual speed has been shown with videogame training in older adults.

Moreover, we speculate that the wider transfer of exergame training that we observed may also be due to other aspects of this training in addition to physical activity or videogame play. Our complex training regimen embodied a combination of aspects including the variability of the tasks and the interactions with another player during the training sessions. The rich environment involved alternation among a wide range of motivating tasks that required several different modes of interaction and varying task difficulty. This training context required the player to alternate between tasks that varied in the priority placed on rapidity (such as the soccer headers game), accuracy (such as the bowling game), strategy (such as the marble game), cognitive judgment (such as the trampoline game) and more global combinations (such as the tennis game). This highly variable environment also engendered physical adjustment to the required activity, either using only the top part of the body, only the lower part of the body, or the whole body. The natural attractiveness of the activities and 
the motivation of individual feedback are other factors that could have aided in learning a new skill or improving a developing one (e.g., Herzog \& Fahle, 1997). An important aspect of what subjects appear to have learned from this training was the ability to distribute their skills among multiple tasks with different processing priorities, and consequently develop the accommodation of their skills to different tasks.

Finally, there was no transfer of training on visuospatial measures. Our results are consistent with those of Basak et al. (2008), who also observed no significant training transfer in older adults on visuospatial tasks with a sedentary strategy game. This absence of transfer could be dependent on the specific types of video games used. Indeed, positive benefits have only been reported with video games that involve "action" even though they are sedentary, such as shooting galleries (Green \& Bavelier, 2003, 2006). Games such as this share a set of qualitative features that may be important for transfer to visuospatial tasks, including extraordinary finger speed and a high degree of perceptual, cognitive, and motor load in the service of an accurate motor plan, unpredictability, and an emphasis on peripheral processing (Green \& Bavelier, 2010). The games that we used in our study did not share these specific characteristics.

One limitation of our study was that we did not have a comparison group that played sedentary videogames or one that engaged only in a social participation or interpersonal interaction program. Hence, we cannot distinguish conclusively between the physical activity component of the training regimen, the cognitive content of the training regimen, and the social stimulation of the regimen as the possible sources of improvement. Many studies have showed that social interaction may longitudinally protect against cognitive decline (e.g., Glei et al., 2005; Zunzunegri, Alvarado, Del Ser, \& Otero, 2003). However, there is no evidence that social interaction produces immediate improvements in cognition. Future research should assess whether the exergames provided an additive effect of physical activity and sedentary 
videogaming, or whether benefits of exergame training reflected an interactive effect of cognitive and physical demand. Clearly, this important direction might permit us to understand more accurately the real potential of this new mode of physical activity.

In summary, our study suggests that exergame training, combining cognitive and physical demands in an intrinsically attractive activity, might be an effective way to promote physical and cognitive improvements among older adults. Indeed, one current health issue is to improve the level of physical activity of elderly adults, particularly with sedentary lifestyle (American College of Sports Medicine, 2006). Thus, exergames could be an entree to vigorous physical exercise and a complement to the conventional physical activity for sedentary older adults. It was encouraging to observe, with physically-simulated sport games, transfer benefits to cognitive and physical skills that are directly involved in functional abilities needed by older adults in everyday living (e.g., Tomaszewski et al., 2009). Indeed, previous studies have demonstrated relationships between neuropsychological performance and everyday function in older adult (e.g., Royall, Palmer, Chiodo, \& Polk, 2004). Several attributes of exergames make them promising for widespread adoption. First, exergame play can be home based, mitigating against environmental barriers to exercise. Second, the games allow for choice among activities, which could lead to sustained play and foster autonomy, and thus result in a personalized intervention for people with diverse physical abilities. Finally, the games are fun and affordable. 


\section{References}

Achtman, R.L., Green, C.S., \& Bavelier, D. (2008). Video games as a tool to train visual skills. Restorative Neurology and Neuroscience, 26, 435-46.

Ackerman, P. L. (1990). A correlational analysis of skill specificity: Learning, abilities, and individual differences. Journal of Experimental Psychology: Learning, Memory, and Cognition, 16, 883-901. doi:10.1037/0278-7393.16.5.883

Ackerman, P. L., \& Cianciolo, A. T. (2000). Cognitive, perceptual speed, and psychomotor determinants of individual differences during skill acquisition. Journal of Experimental Psychology: Applied, 6, 259-290. doi:10.1037/1076-898X.6.4.259

Ackerman, P.L., Kanfer, R., \& Calderwood, C. (2010). Use it or lose it? Wii brain exercise practice and reading for domain knowledge. Psychology and Aging, 25, 753-66. doi:10.1037/a0019277

American College of Sports Medecine (2006). ACSM'S Guidelines for exercise testing and prescription seventh edition. Philadelphia: Lippincott Williams \& Wilkins.

Angeraven, M., Aufdemkampe, G., Verhaar, H.J., Aleman, A., \& Vanhees, L. (2008).

Physical activity and enhanced fitness to improve cognitive function in older people without known cognitive impairment. Cochrane Database of systematic review (Online), 16.

Basak, C., Boot, W. R., Voss, M. W., \& Kramer, A. F. (2008). Can training in a real-time strategy videogame attenuate cognitive decline in adults? Psychology and Aging, 23, 765-77. doi:10.1037/a0013494

Blaskewicz Boron, J., Turiano, N. A., Willis, S. L., \& Schaie, K.W. (2007). Effects of cognitive training on change in accuracy in inductive reasoning ability. Journal of Gerontology: Psychological Sciences, 62, 179-86. 
Bohnen, N., Jolles, J., \& Twijnstra, A. (1992). Modification of the Stroop Color Word Test improves differentiation between patients with mild head injury and matched controls. The Clinical Neuropsychologist, 6, 178-84. doi:10.1080/13854049208401854

Boot, W.R., Kramer, A.F., Simons, D.J., Fabiani, M., \& Gratton, G. (2008). The effects of video game playing on attention, memory, and executive control. Acta psychological, 123, 387-98.

Borg, G.A. (1982). Psychophysical bases of perceived exertion. Medicine and science in sports and exercise, 14, 377-81. doi:10.1249/00005768-198205000-00012

Bovend' eerdt, J., Kemper, H.C.G.,\& Verschuur, R. (1980). MOPER fitnesstest: Handleiding en Prestatieschalen (12-18 jarigen). Haarlem: De Vrieseborch.

Corrigan, J.D., \& Hinkeldey, N.S. (1987). Relationships between parts A and B of the Trail Making Test. Journal of Clinical Psychology, 43, 402-9. doi:10.1002/10974679(198707)43:4<402

Chao, D., Capri, G. F., \& Farmer, D. (2000). Exercise adherence among older adults: Challenges and strategies. Controlled Clinical Trials, 21(5, Supplement 1), 212-7. doi:10.1016/S0197-2456(00)00081-7

Chatelois, J, Van Der Linden, M., Rouleau, N., De Courcy, R., Crépeau, F., \& Malenfant, A (1996). Stroop Flexibilité-4 couleurs. (unpublished data).

Chodzko-Zajko, W.J. (1991). Physical fitness, cognitive performance, and aging. Medicine and science in sports and exercise, 23, 868-72. doi:10.1249/00005768-19910700000016

Clark, J. E., Lanphear, A. K., \& Riddick, C. C. (1987). The effects of videogame playing on the response selection processing of elderly adults. Journal of Gerontology, 42, 82-5. 
Clarkson-Smith, L., \& Hartley, A. A. (1989). Relationships between physical exercise and cognitive abilities in older adults. Psychology and Aging, 4, 183-9. doi:10.1037/08827974.4.2.183

Cobb, B.B., \& Mathews, J.J. (1972). A proposed new test for aptitude screening of air traffic controller applicants (FAA-AM-72-18). Washington, DC: U.S. Department of Transportation, Federal Aviation Administration.

Cohen, J. (1988). Statistical power analysis for the behavioral science (2nd ed.). Hillsdale, NJ: Erlbaum.

Colcombe, S. J., \& Kramer, A. F. (2003). Fitness effects on the cognitive function of older adults: A meta-analytic study. Psychological Science, 14, 125-30. doi:10.1111/14679280.t01-1-01430

Colcombe, S.J., Kramer, A. F., McAuley, E., Erikson, K. I., \& Scalf, P. (2004). Neurocognitive aging and cardiovascular fitness: recent findings and future directions. Journal of molecular neuroscience, 24, 9-14. doi:10.1385/JMN:24:1:009

Craik, F. I. M., \& Salthouse, T. A. (Eds.), (1999). Handbook of aging and cognition (2nd ed.). Hillsdale, NJ: Erlbaum.

Drew, D., \& Waters, J. (1986). Video games: Utilization of a novel strategy to improve perceptual motor skills and cognitive functioning in the non-institutionalized elderly. Cognitive Rehabilitation, 4, 26-31.

Dustman, R. E., Emmerson, R. Y., Steinhaus, L. A., Shearer, D. E., \& Dustman, T. J. (1992). The effects of videogame playing on neuropsychological performance of elderly individuals. Journal of Gerontology, 47, 168-71.

Dustman, R. E., Ruhling, R. O., Russell, E. M., Shearer, D. E., Bonekat, W., Shigeoka, J. W., Wood, J. S., \& Bradford, D. C. (1984). Aerobic exercise training and improved 
neurophysiological function of older adults. Neurobiology of Aging, 5, 35-42. doi:10.1016/0197-4580(84)90083-6

Ekstrom, R. B., French, J. W., Harman, H. H., \& Dermen, D. (1976). Manual for the kit of factor-referenced cognitive tests. Princeton, NJ: Educational Testing Service.

Erickson, K. I., \& Kramer, A.F. (2009). Aerobic exercise effects on cognitive and neural plasticity in older adults. British Journal of Sports Medicine, 43, 22-4. doi:10.1136/bjsm.2008.052498

Etnier, J.L, Nowell, P., Landers, D.M., \& Sibley, B.A. (2006). A meta-regression to examine the relationship between aerobic fitness and cognitive performance. Brain Research Reviews, 52, 119-30. doi:10.1016/j.brainresrev.2006.01.002

Flew, T., \& Humphreys, S. (2005). Games: Technology, industry, culture. Oxford University Press.

Folstein, M.F., Robins, L.N, \& Helzer, J.E. (1983). The Mini-Mental State Examination. Archives of general psychiatry, 40, 812.

Glei, D.A., Landau, D.A., Goldman, N., Chuang, Y.L., Rodríguez, G., \& Weinstein, M. (2005). Participating in social activities helps preserve cognitive function: an analysis of a longitudinal, population-based study of the elderly. International journal of epidemiology, 34, 864-71. doi:10.1093/ije/dyi049

Goldstein, J., Cajko, L., Oosterbroek, M., Michielsen, M., Van Houten, O., \& Salvedera, F. (1997). Videogames and the elderly. Social Behavior and Personality, 25, 345-52. doi:10.2224/sbp.1997.25.4.345

Graves, L.E., Ridgers, N.D., Williams, K., Stratton, G., Atkinson, G., \& Cable, N.T. (2010). The physiological cost and enjoyment of Wii Fit in adolescents, young adults, and older adults. Journal of Physical Activity and Health, 7, 393-401. 
Green, C. S., \& Bavelier, D. (2003). Action video games modify visual selective attention. Nature, 423, 534-37. doi:10.1038/nature01647

Green, C. S., \& Bavelier, D. (2006). Effects of action video game playing on the spatial distribution of visual selective attention. Journal of Experimental Psychology: Human Perception and Performance, 32, 1465-78. doi:10.1037/0096-1523.32.6.1465

Green, C. S., \& Bavelier, D. (2008). Exercising Your Brain: A Review of Human Brain Plasticity and Training-Induced Learning. Psychology and Aging, 23, 692-701. doi:10.1037/a0014345

Green, C. S., Li, R., \& Bavelier, D. (2010). Perceptual learning during action video game playing. Topics in Cognitive Science, 2, 202-216. doi:10.1111/j.17568765.2009.01054

Guderian, B., Borreson, L.A., Sletten, L.E., Cable, K., Stecker, T.P., Probst, M.A., Dalleck, L.C. (2010). The cardiovascular and metabolic responses to Wii Fit video game playing in middle-aged and older adults. The Journal of Sports Medicine and Physical Fitness, 50, 436-42.

Hawkins, H.L, Kramer, A.F,\& Capaldi, D. (1992). Aging, exercise and attention. Psychology and Aging, 7, 643-53. doi:10.1037/0882-7974.7.4.643

Hedges, L.V. (1982). Estimation of effect size from a series of independent experiments. Psychological Bulletin, 92, 490-9. doi:10.1037/0033-2909.92.2.490

Herzog, M.H., \& Fahle, M. (1997). The role of feedback in learning a vernier discrimination task. Vision research, 37, 2133-41. doi:10.1016/S0042-6989(97)00043-6

Hillman, C.H., Motl, R.W., Pontifex, M.B., Posthuma, D., Stubbe, J.H., Boomsma, D.I., \& de Geus, E.J.C. (2006). Physical activity and cognitive function in a cross-section of younger and older community-dwelling individuals. Health Psychology, 25, 678-87. doi:10.1037/0278-6133.25.6.678 
Holm, S. (1979). A simple sequentially rejective multiple test procedure. Scandinavian Journal of Statistics, 6, 65-70.

Howley, E.T. (2001). Type of activity: Resistance, aerobic and leisure versus occupational physical activity. Medicine and Science in Sports and Exercise, 33, 364-69. doi:10.1097/00005768-200106001-00005

Hoyer, W. J., \& Verhaeghen, P. (2006). Memory aging. In J. Birren \& W. Schaie (Eds.), Handbook of the psychology of aging (pp. 209-232). Burlington, MA: Elsevier Academic Press. doi:10.1016/B978-012101264-9/50013-6

Hughes, S.L., Seymour, R.B., Campbell, R.T., Whitelaw, N., \& Bazzarre, T. (2009). Bestpractice physical activity programs for older adults: Findings from the national impact study. American Journal of Public Health, 99, 362-8. doi:10.2105/AJPH.2007.131466

Hultsch, D. F., Hertzog, C., Small, B.J, \& Dixon, R.A. (1999). Use it or lose it: engaged lifestyle as a buffer of cognitive decline in aging? Psychology and Aging, 14, 245-63. doi:10.1037/0882-7974.14.2.245

Jones, C., \& Hammig, B. (2009). Case report: Injuries associated with interactive game consoles: preliminary data. The Physician and Sportsmedicine, 37, 138-40. doi:10.3810/PSM.2009.04.1695

Kramer, A.F., Bherer, L., Colcombe, S.J., Dong, W., \& Greenough, W.T. (2004). Environmental influences on cognitive and brain plasticity during aging. The Journals of Gerontology. Series A, Biological Sciences and Medical Sciences, 59, 940-57.

Kramer, A. F., Hahn, S., Cohen, N. J., Banich, M. T., McAuley, E., Harrison, C. R., Chason, J., Vakil, E., Bardell, L., Boileau, R. A., \& Colcombe, A. (1999). Ageing, fitness and neurocognitive function. Nature, 400, 418-19. doi:10.1038/22682 
Kriska, A. M., Knowler, W. C., LaPorte, R. E., Drash, A. L., Wing, R. R., Blair, S. N., et al. (1990). Development of questionnaire to examine relationship of physical activity and diabetes in Pima Indians. Diabetes Care, 13, 401-11. doi:10.2337/diacare.13.4.401

Nitz, J.C., Kuys, S., Isles, R., \& Fu, S. (2010). Is the Wii Fit a new-generation tool for improving balance, health and well-being? A pilot study. Climacteric: The Journal of the International Menopause Society, 13, 487-91.

Park, D. C., \& Gutchess, A. H. (2002). Aging, cognition, and culture: a neuroscientific perspective. Neuroscience and Biobehavioral Reviews, 26, 859-67. doi:10.1016/S0149-7634(02)00072-6

Perrot, A., Gagnon, C., \& Bertsch, J. (2009). Physical activity as a moderator of the relationship between aging and inductive reasoning. Research Quarterly for Exercise and Sport, 80, 393-7.

Quiroga, M.A., Herranz, M., Gomez-Abad, M., Kebir, M., Ruiz, J., \& Colom, R. (2009). Video-games: Do they require general intelligence? Computers \& Education, 53, 4148. doi:10.1016/j.compedu.2009.02.017

Rikli, R., \& Jones, J. (1999). Development and validation of a functional fitness test for community-residing older adults. Journal of Aging and Physical Activity, 7, 129-61.

Rikli, R., \& Jones, C.J. (2001). Senior Fitness Test Manual. Champaign, IL: Human Kinetics

Robergs, R., \& Landwehr, R. (2002). The surprising history of the "HRmax=220-age" equation. Journal of Exercise Physiology, 5, 1-10.

Rosenberg, D., Depp, C.A., Vahia, I.V., Reichstadt, J., Palmer, B.W., Kerr., J, Norman, G., \& Jeste, D.V. (2010). Exergames for subsyndromal depression in older adults: A pilot study of a novel intervention. American Association for Geriatric Psychiatry, 18, 2216. doi:10.1097/JGP.0b013e3181c534b5 
Royall, D.R., Palmer, R., Chiodo, L.K., \& Polk, M.J. (2004). Declining executive control in normal aging predicts change in functional status: the Freedom House Study. Journal of the American Geriatrics Society, 52, 346-52. doi: 10.1111/j.15325415.2004.52104.x

Salthouse, T.A. (2004). What and When of Cognitive Aging. Current Directions in Psychological Science, 13, 140-4. doi: 10.1111/j.0963-7214.2004.00293.xShepard, R.N., \& Metzler, J. (1971). Mental rotation of three dimensional objects, Science, 171, 7013. doi:10.1126/science.171.3972.701

Schutzer, K.A., \& Graves, B.S. (2004). Barriers and motivations to exercise in older adults. Preventive medicine, 39, 1056-61. doi:10.1016/j.ypmed.2004.04.003

Shay, K. A., \& Roth, D. L. (1992). Association between aerobic fitness and visuospatial performance in healthy older adults. Psychology and Aging, 7, 15-24. doi:10.1037/0882-7974.7.1.15

Spence, I., \& Feng, J. (2010). Video games and spatial cognition. Review of General Psychology, 14, 92-104. doi:10.1037/a0019491

Tomaszewski, S., et al. (2009). Longitudinal Changes in Memory and Executive Functioning are Associated with Longitudinal Change in Instrumental Activities of Daily Living in older adults. The Clinical Neuropsychologist, 23, 446-461.

doi:10.1080/13854040802360558

U.S. Department of Health and Human Services. (1996). Physical activity and health: A report of the Surgeon General. Pittsburgh, PA: Government Printing Office.

Vance, D.E., McNees, P., \& Meneses, K. (2009). Technology, cognitive remediation, and nursing: Directions for successful cognitive aging. Journal of Gerontological Nursing, 35, 50-6. doi:10.3928/00989134-20090201-09 
Vandenberg, S.G., \& Kuse, A.R. (1978). Mental rotations, a group test of three dimensional spatial visualization, Perceptual and Motor Skills, 47, 599-604.

Wechsler, D. (1997a). Wechsler Adult Intelligence Scale-3rd Edition (WAIS-3R) San Antonio, TX: Harcourt Assessment.

Wechsler, D. (1997b). Wechsler Memory Scale - $3^{\text {rd }}$ Edition (WMS-III). San Antonio, TX: Psychological Corporation.

Wechsler, D. (1999). Wechsler Abbreviated Scale of Intelligence (WASI). San Antonio, TX: Harcourt Assessment.

West, R. (1996). An application of prefrontal cortex function theory to cognitive aging. Psychological Bulletin, 120, 272-92. doi:10.1037/0033-2909.120.2.272

Yesavage, J.A., Brink, T.L., Rose, T.L, Lum, O., Huang, V., Adey M., \& Leirer, V.O. (19821983). Development and validation of a geriatric depression screening scale: a preliminary report. Journal of Psychiatric Research, 17, 37-49. doi:10.1016/00223956(82)90033-4

Zunzunegui, M.V., Alvarado, B.E., Del Ser, T., \& Otero, A. (2003). Social networks, social integration, and social engagement determine cognitive decline in communitydwelling Spanish older adults. The Journals of Gerontology. Series B, Psychological Sciences and Social Sciences, 58, 93-100. 
Authors' Footnote

Pauline Maillot, Alexandra Perrot, CIAMS EA 4532, Université Paris-Sud

Alan Hartley, Scripps College, Claremont, California.

Correspondence concerning this article should be addressed to Pauline Maillot, UFR STAPS

- Bât 335, Université Paris-Sud, 91405 Orsay Cedex, France, pauline.maillot@u-psud.fr. 
Footnote

${ }^{1}$ Chair stand: Number of full stands that can be completed in 30 seconds with arms folded across chest. Arm curl: Number of bicep curls that can be completed in 30 seconds holding a hand weight of $5 \mathrm{lbs}(2.27 \mathrm{~kg})$ for women; $8 \mathrm{lbs}(3.63 \mathrm{~kg})$ for men. 6-Minute Walking Test: The number of meters walked in $6 \mathrm{~min}$, the maximum heart rate, the mean heart rate, and the rated exertion using the Borg scale were recorded. Chair sit and reach: From a sitting position at front of chair, with leg extended and hands reaching toward toes, the number of inches $(\mathrm{cm})(+$ or -$)$ between extended fingers and tip of toe. Back scratch: With one hand reaching over the shoulder and one up the middle of the back, the number of inches (cm) between extended middle fingers (+ or -). 8 foot up and go test: Number of seconds required to get up from a seated position, walk 8 feet $(2.44 \mathrm{~m})$, turn, and return to seated position.

${ }^{2}$ The Letter Sets test and the Matrix Reasoning test are classified as tests of inductive reasoning (e.g., Ackerman, 1990). Researchers have demonstrated that inductive reasoning is a higher executive function important for everyday functioning and sensitive to age-related deficits (e.g., Blaskewicz Boron, Turiano, Willis, \& Schaie, 2007). 
Table 1

Means of Demographic, Health, Exercise, and Subjective Well-Being Variables for Control and Training Groups

\begin{tabular}{|c|c|c|c|c|c|c|}
\hline \multirow[b]{2}{*}{ Variables } & \multicolumn{2}{|c|}{ Control } & \multicolumn{2}{|c|}{ Training } & \multirow[b]{2}{*}{$t$} & \multirow[b]{2}{*}{$p$} \\
\hline & $M$ & $S D$ & $M$ & $S D$ & & \\
\hline Age (years) & 73.47 & 3.00 & 73.47 & 4.10 & 0.00 & 0.81 \\
\hline Self-rated health & 3.67 & 0.62 & 3.73 & 0.59 & 0.30 & 0.76 \\
\hline Years of education & 11.40 & 2.22 & 11.20 & 1.78 & -0.27 & 0.78 \\
\hline Body Mass Index $\left(\mathrm{kg} / \mathrm{m}^{2}\right)$ & 27.62 & 5.89 & 27.40 & 4.46 & -0.18 & 0.90 \\
\hline MMSE & 29.27 & 0.88 & 28.67 & 1.17 & -1.58 & 0.12 \\
\hline GDS & 5.93 & 2.66 & 6.00 & 3.48 & 0.06 & 0.95 \\
\hline MAQ (hr/week) & 0.60 & 0.58 & 0.79 & 0.59 & 0.88 & 0.38 \\
\hline
\end{tabular}

Note. $M=$ Mean. $S D=$ Standard Deviation. $M M S E=$ Mini Mental State Examination. GDS = Geriatric Depression Scale. $M A Q=$ Modifiable Activity Questionnaire 
Table 2

\section{Comparison on Pre-test Measures by Condition}

\begin{tabular}{|c|c|c|c|c|c|c|}
\hline & \multicolumn{2}{|c|}{ Control } & \multicolumn{2}{|c|}{ Training } & \multirow[b]{2}{*}{$t$} & \multirow[b]{2}{*}{$p$} \\
\hline & $M$ & $S D$ & $M$ & $S D$ & & \\
\hline Resting Heart Rate (bpm) & 63.60 & 8.08 & 64.57 & 8.33 & -0.32 & 0.75 \\
\hline Max Heart Rate 6-min Walk (bpm) & 107.87 & 16.02 & 116.57 & 17.45 & -1.40 & 0.17 \\
\hline Mean Heart Rate 6-min Walk (bpm) & 100.27 & 15.10 & 106.86 & 11.63 & -1.31 & 0.20 \\
\hline Chair stands (Number) & 14.27 & 3.67 & 12.93 & 2.66 & 1.14 & 0.26 \\
\hline Arm curls (Number) & 16.80 & 2.57 & 16.53 & 3.25 & 0.25 & 0.80 \\
\hline 6-min Walk (Meters covered) & 429.84 & 61.58 & 411.17 & 84.69 & 0.69 & 0.50 \\
\hline 8-foot Up-and-Go (sec) & 7.12 & 1.64 & 7.42 & 1.38 & -0.56 & 0.58 \\
\hline Back Scratch "lower right" $(\mathrm{cm})$ & 0.87 & 6.17 & 3.67 & 8.17 & -1.06 & 0.30 \\
\hline Back Scratch "lower left" (cm) & 0.90 & 6.74 & 3.40 & 8.73 & -0.88 & 0.39 \\
\hline Back Scratch "upper right" (cm) & 5.57 & 9.08 & 5.63 & 8.29 & -0.02 & 0.98 \\
\hline Back Scratch "upper left" (cm) & 14.43 & 13.51 & 13.03 & 10.85 & 0.31 & 0.76 \\
\hline Perceived Effort 6-min Walk & 2.87 & 1.06 & 4.00 & 1.47 & -2.40 & 0.02 \\
\hline
\end{tabular}

Note. $M=$ Mean. $S D=$ Standard Deviation. 
Table 3

Comparison of Pretest-Posttest Change Scores on Physical Measures for Training and Control Groups

\begin{tabular}{lccccccc}
\hline & \multicolumn{1}{c}{ Control } & \multicolumn{1}{c}{ Training } & & & \\
\cline { 2 - 8 } & $M$ & $S D$ & $M$ & $S D$ & $t$ & $p$ & $\eta^{2}$ \\
\hline Physical Measures & & & & & & & 0.693 \\
Max Heart Rate 6-min Walk (bpm) & -4.07 & 10.15 & 10.50 & 15.92 & -2.96 & $<0.01$ & 0.227 \\
Mean Heart Rate 6-min Walk (bpm) & -3.80 & 11.62 & 7.43 & 13.52 & -2.40 & 0.01 & 0.169 \\
Chair Stands (Number) & -1.07 & 1.94 & 2.73 & 2.28 & -4.91 & $<0.01$ & 0.528 \\
Arm Curls (Number) & 0.00 & 2.73 & 3.00 & 2.70 & -3.02 & $<0.01$ & 0.262 \\
6-min Walk (Meters covered) & 3.07 & 26.54 & 58.05 & 40.45 & -4.40 & $<0.01$ & 0.408 \\
8-foot Up and Go (sec) & 0.48 & 1.05 & -0.94 & 0.62 & 4.53 & $<0.01$ & 0.424 \\
Back Scratch "lower right" (cm) & 2.00 & 5.80 & -2.78 & 6.20 & 2.18 & 0.02 & 0.130 \\
Back Scratch "lower left" (cm) & 2.10 & 6.88 & -2.22 & 6.27 & 1.80 & 0.04 & 0.107 \\
Back Scratch "upper right" (cm) & -3.85 & 10.35 & -0.50 & 5.33 & -1.12 & 0.14 & 0.051 \\
Back Scratch "upper left" (cm) & -9.40 & 18.04 & 0.25 & 3.90 & -2.03 & 0.03 & 0.017 \\
\hline Perceived Exertion (Borg Scale) & 0.67 & 1.11 & 0.64 & 1.82 & .043 & 0.48 & 0.001
\end{tabular}

Note. $M=$ Mean. $S D=$ Standard Deviation. $\eta^{2}=$ effect size (Cohen, 1988). For heart rate, chair stand and arm curl, a positive mean corresponds to an improvement between pre-and post-test. For 8 foot Up and Go, and back scratch, a negative mean corresponds to an improvement between pre-and post-test. All p values smaller than $p=.05$ remain significant after Bonferroni correction. 
Table 4

Comparison of Pretest-Posttest Change Scores on Executive Function, Processing Speed, and Visuospatial Measures for Training and Control Groups

\begin{tabular}{|c|c|c|c|c|c|c|c|}
\hline \multirow[b]{2}{*}{ Tasks } & \multicolumn{2}{|c|}{ Control } & \multicolumn{2}{|c|}{ Training } & \multirow[b]{2}{*}{$t$} & \multirow[b]{2}{*}{$p$} & \multirow[b]{2}{*}{$\eta^{2}$} \\
\hline & $M$ & $S D$ & $M$ & $S D$ & & & \\
\hline Executive Function & & & & & & & 0.805 \\
\hline Trail Making Test Part B - Part A (sec) & 3.70 & 28.54 & -15.42 & 20.27 & -2.12 & 0.04 & 0.138 \\
\hline Stroop: Incongruent (Number) & 0.60 & 4.05 & 9.13 & 8.80 & -3.412 & $<0.01$ & 0.294 \\
\hline Stroop: Task Switching (Number) & 0.27 & 4.68 & 8.27 & 5.73 & -4.189 & $<0.01$ & 0.385 \\
\hline Letter Sets (Number) & 0.07 & 1.03 & 1.87 & 1.85 & -3.30 & $<0.01$ & 0.279 \\
\hline Matrix Reasoning Test (Number) & -0.53 & 3.02 & 4.20 & 1.21 & -5.636 & $<0.01$ & 0.531 \\
\hline Digit-Symbol Substitution (Number) & 0.20 & 5.65 & 9.07 & 7.97 & -3.516 & $<0.01$ & 0.306 \\
\hline Visuospatial & & & & & & & 0.230 \\
\hline Spatial Span Test (Number) & -0.27 & 1.28 & 0.33 & 1.40 & -1.226 & 0.12 & 0.051 \\
\hline Backwards Spatial Span Test (Number) & 0.27 & 0.96 & 0.07 & 1.67 & 0.402 & 0.35 & 0.006 \\
\hline Direction Headings (Number) & -0.67 & 3.22 & 2.13 & 3.68 & -2.217 & 0.02 & 0.149 \\
\hline Mental Rotation (Number) & -0.20 & 2.35 & 0.40 & 2.17 & -.726 & 0.24 & 0.019 \\
\hline Processing Speed & & & & & & & 0.788 \\
\hline \multicolumn{8}{|l|}{ Perceptual Speed } \\
\hline Cancellation (Number) & -2.53 & 6.56 & 10.00 & 6.09 & -5.423 & $<.01$ & 0.512 \\
\hline Number Comparison (Number) & -0.73 & 2.02 & 2.87 & 2.72 & -4.116 & $<.01$ & 0.377 \\
\hline \multicolumn{8}{|l|}{ Psychomotor Speed } \\
\hline Movement Time: Simple RT (ms) & 21.59 & 64.55 & -38.55 & 74.36 & 2.365 & .03 & 0.166 \\
\hline Movement Time: Choice RT (ms) & 21.84 & 57.16 & -42.75 & 67.24 & 2.835 & $<.01$ & 0.223 \\
\hline Decision Time: Simple RT (ms) & 30.98 & 92.77 & -103.07 & 92.57 & 3.962 & $<.01$ & 0.359 \\
\hline Decision Time: Choice RT (ms) & -16.26 & 81.74 & -104.15 & 74.28 & 3.082 & $<.01$ & 0.253 \\
\hline Tapping (Right Hand, Number) & -0.53 & 3.75 & 7.00 & 6.81 & -3.755 & $<.01$ & 0.335 \\
\hline Tapping (Left Hand, Number) & -0.80 & 3.37 & 5.10 & 6.03 & -3.306 & $<.01$ & 0.281 \\
\hline
\end{tabular}

Note. $M=$ Mean. $S D=$ Standard Deviation. $\eta^{2}=$ effect size (Cohen, 1988). For timed tests, a negative mean corresponds to an improvement between pre-and post-test. For scored tests (indicated by Number), a positive mean corresponds to an improvement between pre-and post-test. All $p$ values smaller than $p=.05$ remain significant after Bonferroni correction. 